\title{
Base Station Energy Efficiency Improvement for Next Generation Mobile Networks
}

\author{
Weston Mwashita and Marcel Ohanga Odhiambo
}

\begin{abstract}
As more and more Base Stations (BSs) are being deployed by mobile operators to meet the ever increasing data traffic, solutions have to be found to try and reduce BS energy consumption to make the BSs more energy efficient and to reduce the mobile networks' operational expenditure (OPEX) and carbon dioxide emissions. In this paper, a BS sleeping technology deployable in heterogeneous networks (HetNets) is proposed. The proposed scheme is validated by using extensive OMNeT++/SimuLTE simulations. From the simulations, it is shown that some lightly loaded micro BSs can be put to sleep in a HetNet when the network traffic is very low without compromising the QoS of the mobile network.
\end{abstract}

Keywords-Energy efficiency, Base Station sleeping, $\mathrm{CO}_{2}$ emissions, heterogeneous networks, mobile networks, next generation networks, Operational Expenditure (OPEX)

\section{INTRODUCTION}

$\mathbf{O}$ F late, mobile broadband services have seen an explosive and unprecedented growth that has triggered an exponential growth in data traffic. CISCO in [1] predicts that smartphone traffic will surpass PC traffic by 2020 and also predicts that 160 Exabytes (160 quintillion bytes) of data per month can be reached by 2020 as shown in Figure 1.

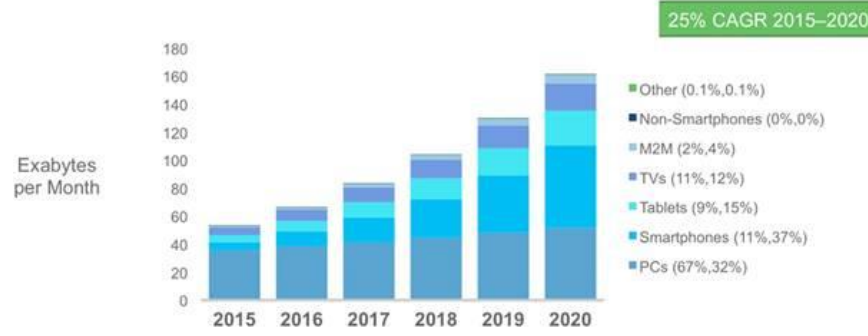

Fig. 1. Global Internet Traffic by Device Type [1]

Mobile operators are responding to this high demand for data by upgrading their mobile networks. They are expanding their networks by adding more and more BSs. In a bid to ensure End-to-End (E2E) Quality of Experience (QoE) of its close to half a billion users, China Mobile, to cite one example, has been doubling its number of BSs annually [2]. These BSs require energy for their normal operation. With this rapid increase in the number of BSs, the energy consumption increases in the same way. The most reliable sources of energy for BSs are the

Weston Mwashita is with the University of South Africa, South Africa (e-mail: 47955864@mylife.unisa.ac.za).

Marcel Ohanga Odhiambo is with Department of Computer Systems and Process Control at Vaal University of Technology, South Africa (e-mail: marcelo@vut.ac.za).

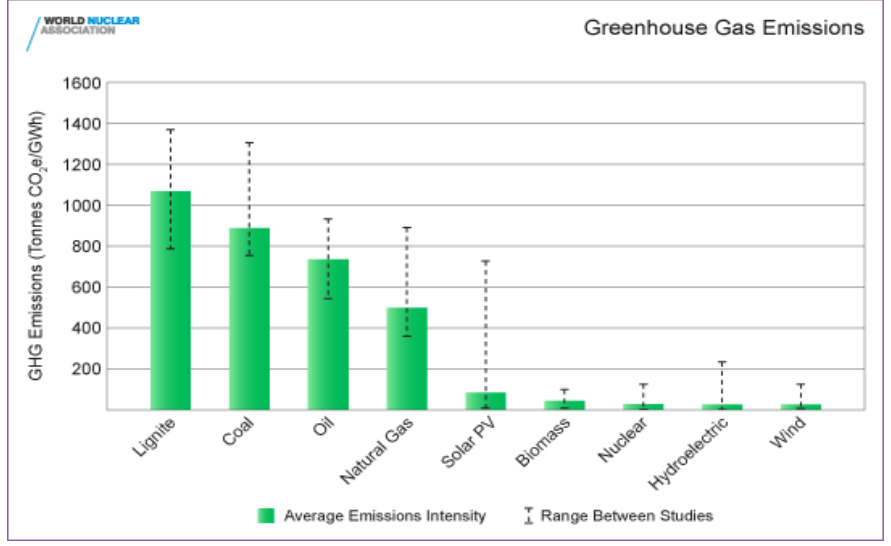

Fig. 2. Greenhouse gas emissions from electricity generation [3]

national electrical grids. Most of the methods that are used to produce this electricity that feed the national grids produce $\mathrm{CO}_{2}$ emissions [3].

Even nuclear generation of electricity results in $\boldsymbol{C O}_{2}$ emissions especially in the enrichment process of uranium. Figure 2 shows the Greenhouse Gas emissions from various electricity production methodologies.

It can be seen from Figure 2 that even the hydro production of electricity indirectly produces $\boldsymbol{C O}_{2}$ emissions. The explosive data growth results in many BSs set up in a cellular network leading to an increased energy consumption levels. A higher demand in energy levels leads to a higher $\boldsymbol{C O}_{2}$ emissions. The emissions result in global warming which in turn leads to disastrous climate changes [4].

Figure 3 shows two EF-4 tornadoes within a mile apart that swept past Pilger in Nebraska on 16 June 2014 [5]. Two people were killed and many infrastructures were damaged in the town.

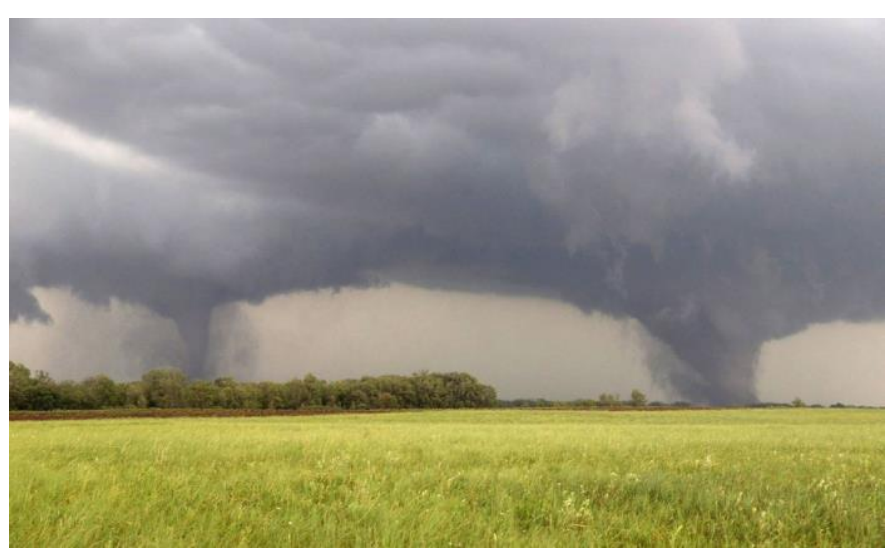

Fig.3. Twin tornadoes approaching Pilger on 16 June 2014 [5] 
Weather experts attributed this unique phenomenon to global warming. In light of these undesirable consequences of $\boldsymbol{C O}_{2}$ emissions, researchers in telecommunications engineering have come up with strategies to reduce the carbon dioxide emissions.

Researchers from industry and academia are in agreement that the next generation mobile networks have to have some form of BS sleeping technologies to save on energy consumption [6], [7], [8], [9], [10]. The exponential mobile traffic growth has not only affected the environment but is also impacting negatively on network operators' expenditure. According to [11], mobile operators have to come up with strategies to deal with the fast growing mobile services by providing these services at an acceptable QoS and QoE whilst enjoying a sound financial performance. As can be seen from Figure 4, mobile operators are facing a dilemma in that the revenue is not growing at the same rate as the traffic growth.

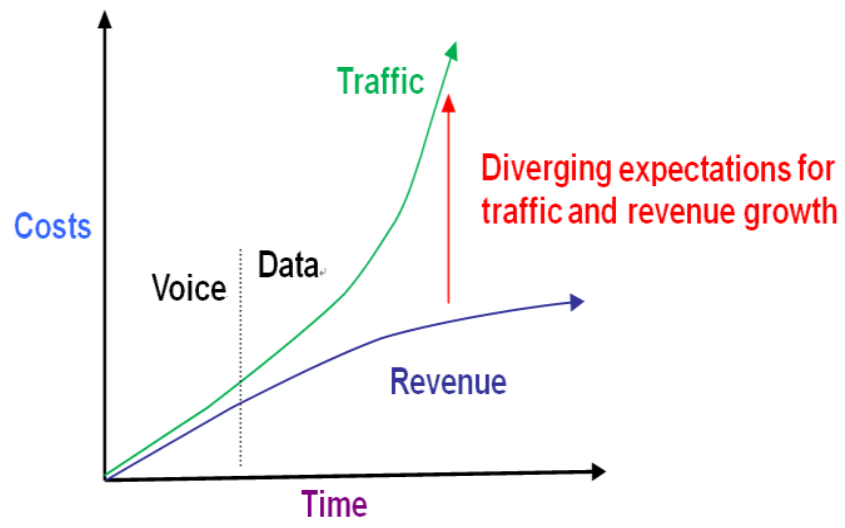

Fig. 4. Variation of mobile operator's OPEX [11]

This is because of the investment cost in the form of capital (CAPEX) and operational (OPEX) expenditures that are not moving in tandem with traffic growth. This means that to increase revenue, mobile operators need to optimize their expenditure. Scientists have estimated that ICT (including mobile cellular systems) contribute 3\% [12] $\boldsymbol{C O}_{2}$ emissions. This $3 \%$ is equivalent to $25 \%$ of all car emissions in the world. [13].

This paper is organized as follows: section I presents the background of the research. Section II presents the objectives of the research. Section III follows up with a brief review of the existing BS sleeping technologies proposed by various researchers. The proposed solution and the simulation study are presented in sections IV and V respectively. The results are presented and discussed in section VI. The paper is concluded in section VII that also outlines applications, implications of the research work and recommendations for further work.

\section{OBJECTIVES OF THE RESEARCH}

This paper aims to develop an algorithm that puts to sleep certain micro BSs when the network traffic is very low and switch them back ON when network traffic reaches a certain threshold. The scheme should increase the energy efficiency of a network without compromising the QoS. The main emphasis of the proposed scheme is on the QoS of the network. Most BS sleeping schemes have put emphasis on energy saving at the expense of QoS. For user equipment (UEs) that have to be transferred to still active BSs so that certain micro BSs can go to sleep, this should be done in an energy efficient manner.

\section{RELATED WORK}

More and more researchers have been drawn into the designing of BS sleeping schemes to increase energy efficiency of BSs in mobile networks. In [6] a scheme called "“Green" Distance Aware Base Station Sleeping Algorithm" is advanced. In this scheme, each BS estimates the distances of all its UEs and also obtains similar UE distance information from its neighbours through the $\mathrm{X} 2$ interface. The BSs then use this information to compute the average distance of the traffic load. The BSs are then ranked in descending order based on the average distance value. The micro BS with the highest average distance value, is switched OFF after ascertaining that the process does not lead to QoS degradation. Neighbouring BSs then have to increase their transmitting powers to cover cells where micro BSs have been put to sleep. Implementing this scheme in HetNets may result in most macro BSs being switched OFF since macros cover larger areas compared to micro BSs. Normally, macro BSs are the ones that provide uninterrupted network coverage with micro BSs addressing issues of capacity. A scheme that results in some macro BSs being put to sleep will surely result in coverage holes. With this scheme, the moment some BSs are switched OFF, the remaining BSs increase their transmitting powers to try and reduce coverage holes. This however has a tendency of reducing the intended energy savings. Interference levels increase because of increased overlapping areas. The scheme does not suggest a solution to deal with this increased interference levels.

Authors in [7] proposed a cell wilting and blossoming scheme. The scheme involves the monitoring of activity of BSs in a cellular network. Once an underutilized BS has been found, a set of neighbouring BSs that are in a position to take over traffic are identified. Upon confirmation of a sleep mode trigger, the underutilized BS slowly reduces its transmitting power whilst neighbouring BSs expand their coverage areas by progressively increasing their transmitting powers. This process is illustrated in Figure 5.

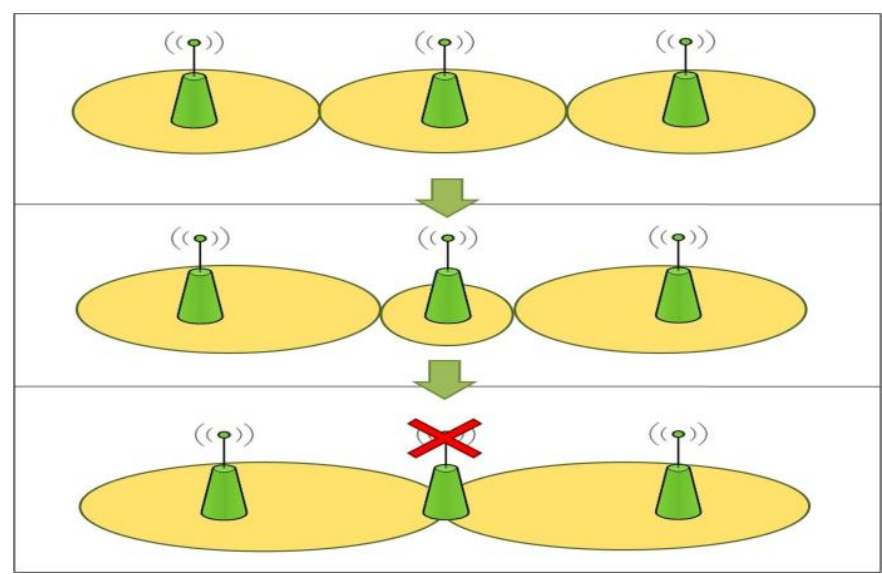

Fig. 5. Example of BS wilting procedure [7]

As neighbouring BSs progressively increase their transmit powers, because of increased areas of overlap, interference levels also increase. The proposed scheme does not address the issue of increased interference and coverage holes upon 
implementation of the cell zooming scheme. The scheme is also silent on the issue of HetNets. The cell wilting and blossoming scheme does not guarantee a continual network coverage. As cells zoom in or out, coverage holes emerge and the authors did not suggest a solution to these coverage holes. The scheme cannot result in reasonable energy savings because as some underutilized $\mathrm{BSs}$ are switched $\mathrm{OFF}$, the remaining $\mathrm{BSs}$ progressively increase their powers to provide adequate coverage. The total increase in power will somehow offset the power reduction that would have resulted from some BSs switching OFF.

Researchers in [8] came up with a heterogeneous energy sources BS sleep control, a scheme where BSs are heterogeneously powered, with some BSs getting power from solar and wind and the others getting power from the national electrical grids. A sophisticated method is however required to coordinate the sleep modes used in this scheme. Problems may be encountered when maximum energy has to be provided at a time when solar or wind powered BSs have limited harvested energy.

SWES (Switching ON/OFF based energy saving scheme) was proposed in [9]. In this scheme, BSs and UEs share signal strength and system load periodically. This information is then used by individual BSs to decide whether to switch OFF or to remain $\mathrm{ON}$. The scheme does not make use of a central controller. BSs use network impact and information from UEs and neighbouring BSs to decide whether to switch OFF or to remain active. The authors defined network impact as, "how much the switching OFF of a particular BS will affect the network". The following factors are used to calculate the network impact:

- Neighbouring BSs’ system load

- Overall traffic profile

- A prescribed threshold

Once a decision to switch OFF has been reached by a BS, it sends a request to switch OFF (RTSO) to its neighbours. This is done to prevent neighbouring BSs with overlapping coverage areas from switching OFF at the same time. A BS can only switch OFF upon receiving confirmation to switch OFF (CTSO) message from all the neighbours. The scheme does not make use of a central controller which means there is strong possibility that the scheme can suffer from network coverage holes. Also in the event that the RTSO and CTSO are exchanged simultaneously, the proposed algorithm might not operate effectively which may end up compromising the QoS of a network. The authors never tested the algorithm on a HetNet which means its applicability with next generation networks is questionable.

In [10], the authors introduced a scheme that does not switch OFF BSs using a least traffic load metric, but by use of the User Equipment's total power. UEs are handed over to BSs that have the highest received power and have to adapt their transmitting power to cater for the altered BS-UE distances. BSs are then ranked according to the total power needed by UEs to connect to still active BSs. The BS that requires the least power for UEs to connect to it is the one that is switched OFF first. The scheme needs to incorporate additional signalling for the acquisition of UEs and BSs locations within the network by a central station. From the extensive simulations carried out by the researchers, they came to the conclusion that the performance of the scheme is better than that of cell zooming technologies especially when it comes to the number of BSs that can be switched OFF as network changes. The authors however, did not investigate how their scheme affects QoS. There is no mention of coverage holes in their scheme though it is obvious that where some BSs have to be switched OFF in a network, the issue of coverage holes needs to be addressed. The authors designed the scheme for $5 \mathrm{G}$ networks but they did not show how the scheme handles HetNets. 5G networks will be heterogeneous in nature.

Other BS sleeping technologies can be found in [15], [16]. For most of these schemes, the main emphasis by the researchers was on energy saving and this resulted in most of them producing high energy savings. The main problem with these schemes is lack of emphasis on QoS. Schemes that may result in huge energy savings but with compromised QoS are not easily taken on board by network operators. When QoS is compromised, not many users are willing to subscribe to such networks. Network operators are aware of this and would not want to risk their businesses just for the sake of reducing $\mathrm{CO}_{2}$ emissions.

\section{THE PROPOSED SOLUTION}

The proposed scheme divides a cellular network into 7 cell clusters. Within the cluster, the BS at the centre becomes the central controller. The controller is responsible for BSs within one cluster and there can be many controllers within a cellular network depending on the number of clusters in the network. A 7 cell cluster is shown in Figure 6.

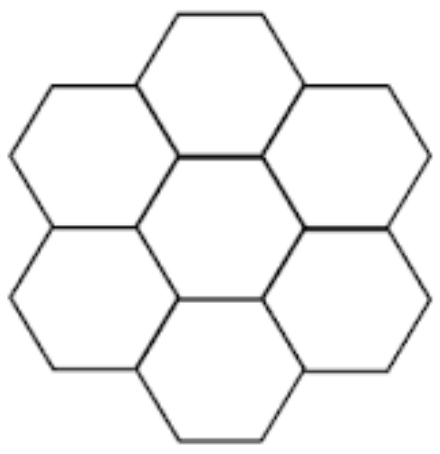

Fig. 6. A 7 cell sector

The 7 cells shown in Figure 6 are all macro cells. Micro cells, pico cells or femto cells are not shown. These small cells are imbedded within the cluster as needed. It is proposed that, to upgrade a cellular network in order to cater for the unprecedented traffic growth, the macros should be left intact. Small cells should then be installed in areas where there are high volumes of traffic within the 7 cell cluster. The macros within the cluster provide uninterrupted cellular coverage to users within the cluster and the micros deal with issues of traffic volumes. The macros should always be left $\mathrm{ON}$ so that all areas within the network are covered. The central controller uses the BS sleeping algorithm to put to sleep lightly loaded micro BSs. This ensures that as cellular traffic increases exponentially, the unavoidable upgrading of the system to cater for the increase is energy efficient.

BSs can only be put to sleep provided this action does not lead to a compromised QoS. The next section presents the pseudocode of the BS sleeping scheme starting with the notations to be used in the pseudocode. 
A. Notations used

TABLE I

NOTATIONS TO BE USED IN THE PSEUDOCODES

\begin{tabular}{|c|l|}
\hline$\alpha_{\text {th }}$ & Normalised traffic threshold \\
\hline$\alpha_{\text {max }}$ & $\begin{array}{l}\text { Maximum normalised traffic load } \\
\text { to achieve a certain demanded QoS }\end{array}$ \\
\hline$\alpha_{\text {load }}$ & Normalised traffic load \\
\hline$\beta_{\text {on }}$ & Active BSs \\
\hline$\beta_{\text {sleep }}$ & BSs in sleep mode \\
\hline$\beta$ & A set of BSs in a sector \\
\hline$\beta_{\text {macro } i}$ & Macro BS i \\
\hline$\beta_{\text {micro } j}$ & Micro BS j \\
\hline$\beta_{\text {neigbouring } i}$ & Neighbouring Macro BS i \\
\hline$\alpha_{\text {load } \rightarrow o}$ & Traffic to be handed over \\
\hline$\alpha_{\text {UE } i}$ & UE i traffic load \\
\hline &
\end{tabular}

\section{B. The BS sleeping pseudocode}

1: Inputs: $\alpha_{t h}, \alpha_{\max }, \beta, \beta_{\text {macro } i,} \beta_{\text {micro } j}$

2: Outputs: $\beta_{\text {on } i}, \beta_{\text {sleep } i}$

3: Initialize $\beta_{\text {on } i} \in \beta$ and $\beta_{\text {sleep } i} \in \beta$

4: Selection sort micro BSs

5: while $\beta_{\text {micro } j}$ 's $\alpha_{\text {load }}<\alpha_{\text {th }}$ do

6: $\quad$ if $\left(\beta_{\text {neigbouring } i}\right.$ 's $\left.\alpha_{\text {load }}+\alpha_{\text {load } \rightarrow 0}\right)<\alpha_{\text {max }}$ then

7: $\quad$ Broadcast intention to switch OFF $\beta_{\text {micro } j}$

8: $\quad$ if pairing is acceptable then

9: $\quad$ Switch OFF $\beta_{\text {micro } j}$

10: $\quad$ end if

11: else terminate process

12: end if

13: end while

As soon as BSs receive a broadcast message of intention to switch OFF certain micro BSs, the neighbouring macro BSs calculate their specific energy. Specific energy (SE) is a metric that is very sensitive to the traffic load being handled by a specific BS [17]. It is the amount of energy that is needed to transport one bit of data from one point to the other. The controller sorts the SEs in descending order. The UEs are also arranged in descending order according to the traffic each UE is handling. A high SE means that the BS is processing low traffic load. This means that the UE that is handling the heaviest load is handed over to the BS processing the lightest load to ensure that a high QoS of service is easily achieved. The next section presents the pseudocode that is used to handover UEs from a micro BS that is switching OFF to a neighbouring macro BS that can take over the traffic in an energy efficient manner.

\section{The BS-UE association pseudocode}

1: Pair BS-UE according to SE and UE traffic load

2: for all BS-UE pairings do

3: if BS can serve UE then

4: Accept handover

5: else terminate process

6: end if

7: end for

\section{THE SIMULATION MODEL}

To validate the proposed algorithms, extensive simulations were carried out using OMNeT++ 4.6. [18]. SimuLTE [19]

simulator for LTE and LTE-A networks, was used to test certain parameters of the proposed network. SimuLTE had to be built on INET 2.3.0 [20]. The experimental set up is shown in Figure 7.

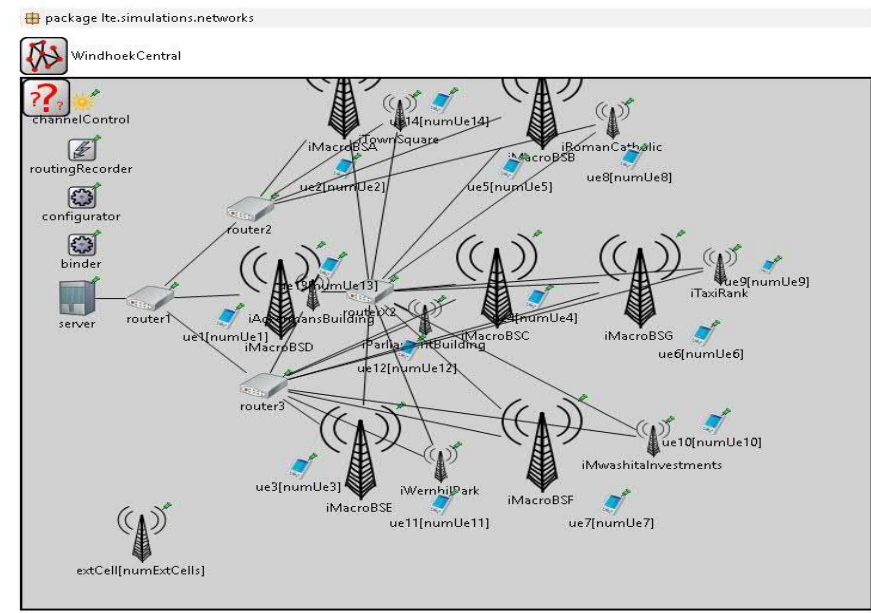

Fig. 7. The experimental set up 0.9 .1 , which is an open source

In the experimental set up, there are seven macro BSs and seven micro BSs. Mobiles are connected to these BSs in such a way that a realistic network is mimicked. Initially, no micro BS was put to sleep. VoIP packets were allowed to circulate in the network and measurements on the following parameters were taken:

- End-to-end delay

- Sum throughputs

- Channel Quality Indicator

- Packet loss

- Total power consumed

The main aim of the simulations was to determine whether it was possible to put to sleep lightly loaded micro BSs in a cellular network and maintain an acceptable QoS. Where a BS is switched OFF, it leaves behind an area that is not covered. These coverage holes affect the QoS of the network.

\section{DISCUSSION OF RESULTS}

\section{A. End-to-end delay}

End-to-end delay is the total delay that is encountered by packets from one end of the network to the other. It is a very important parameter that is used to monitor performance of mobile networks. If end-to-end delay is too high, real time applications cannot be supported by mobile networks and as a result, potential subscribers of the network will refrain from using the network. It was important to check the effect of the BS sleeping scheme on the end-to-end delay. End-to-end delay was measured before certain micro BSs were put to standby and measured again after putting certain micro BSs to sleep. Table II shows these results and the results are plotted in Figure 8. 
It can be seen from these results that the end-to-end delay that was experienced before certain micro BSs were put to sleep suffered very small changes. This means that the QoS of the network was not affected by the introduction of the BS sleeping scheme since the end-to-end delay of the VoIP packets did not change that much. The very small changes observed could have been due to the change in the paths taken by the VoIP packets when some micro BSs are put to sleep.

TABLE II

END-to-end Delay in Seconds Before and After Some BSs Were SWITCHED OFF

\begin{tabular}{|l|l|l|}
\hline UE & $\begin{array}{l}\text { End-to-end delay before } \\
\text { some BSs were switched } \\
\text { OFF }\end{array}$ & $\begin{array}{l}\text { End-to-end delay after } \\
\text { some BSs were switched } \\
\text { OFF }\end{array}$ \\
\hline 1 & 0.00488 & 0.004977 \\
\hline 2 & 0.00482 & 0.004819 \\
\hline 3 & 0.00482 & 0.004819 \\
\hline 4 & 0.00476 & 0.004797 \\
\hline 5 & 0.0047 & 0.00488 \\
\hline 6 & 0.00464 & 0.004977 \\
\hline 7 & 0.00464 & 0.00488 \\
\hline 8 & 0.0057 & 0.005819 \\
\hline 9 & 0.00476 & 0.00476 \\
\hline 10 & 0.00476 & 0.0048 \\
\hline 11 & 0.004593 & 0.004598 \\
\hline 12 & 0.004626 & 0.004638 \\
\hline 13 & 0.00483 & 0.004836 \\
\hline 14 & 0.0048 & 0.0048 \\
\hline 15 & 0.00491 & 0.00499 \\
\hline 16 & 0.004816 & 0.004829 \\
\hline 17 & 0.004793 & 0.00482 \\
\hline
\end{tabular}

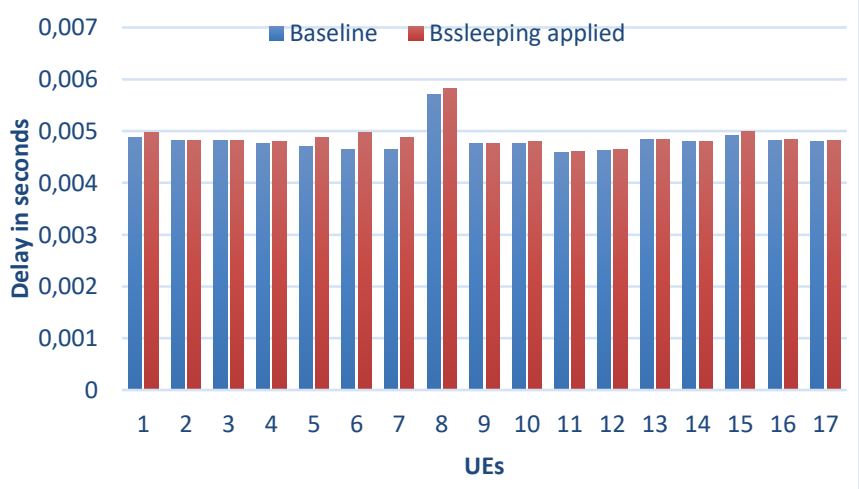

Fig. 8. Mean VoIP end-to-end delay for 17 UEs

\section{B. Packet Loss}

A network that drops too many packets may not be good enough to host audio and video applications. If packet losses exist in the network, they should be very minimum. For this research, the packet loss rate was measured before some micro BSs were put to sleep and measured again after some micro BSs had been put to sleep. As expected, no single packet was dropped before and after certain micro BSs were put to sleep. This was largely expected because the traffic generated before and after some micro BS were put to sleep was very low. Figure 9 shows the normalized traffic for an urban setting. With a normalized traffic of less than 0.3 , the macro BSs have the capacity to cater for all the traffic without dropping any packet.

The BS sleeping scheme is only activated when cellular traffic is very low in the network. It is therefore highly possible for no packets to be dropped when some micro BSs are switched OFF because at this time, traffic will be very low.

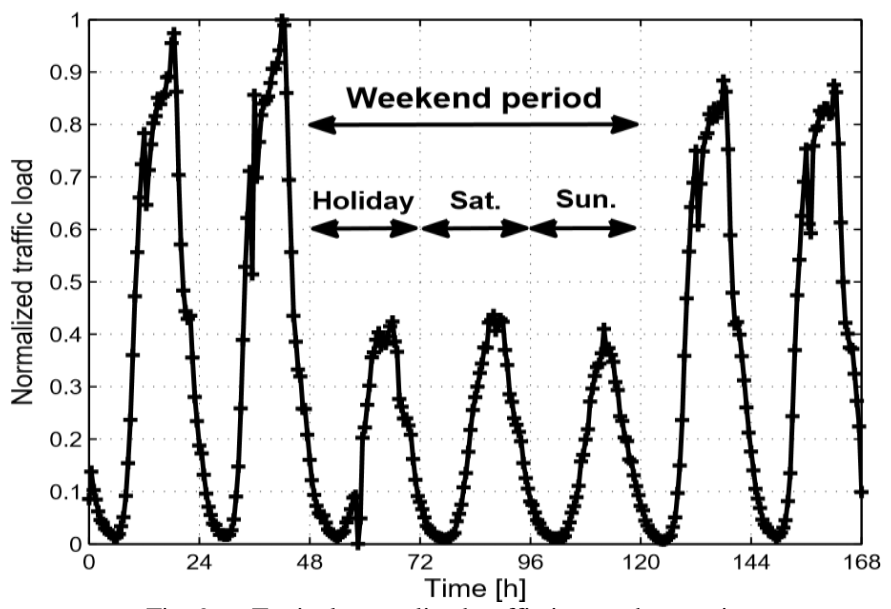

Fig. 9: Typical normalized traffic in an urban setting

\section{Queue Length}

The QoS of a network can ascertained by measuring queue length at certain network elements within a network. For a queueing system, packets queue up, waiting for a service at a particular node. Long queues will lead to some packets being dropped. For this research, VoIP packets were generated and forwarded to a server that responded by sending back the VoIP packets. Queue length at the main server before some micro BSs were switched OFF was measured and measured again after switching some micro BSs OFF. Table III and Figure 10 show the results that were obtained.

Table III:

QUeUe LeNGTH IN SEC. AFTER AND BEFore SWITCHING OFF SOME MICRO BSS

\begin{tabular}{|l|l|l|}
\hline $\begin{array}{l}\text { Simulation } \\
\text { time in sec. }\end{array}$ & $\begin{array}{l}\text { Queue length before } \\
\text { switching OFF some BSs }\end{array}$ & $\begin{array}{l}\text { Queue length after } \\
\text { switching OFF some BSs }\end{array}$ \\
\hline 0.00005 & 9 & 10 \\
\hline 0.00011 & 7 & 8 \\
\hline 0.00017 & 4 & 5 \\
\hline 0.00023 & 3 & 3 \\
\hline 0.00029 & 3 & 3 \\
\hline
\end{tabular}

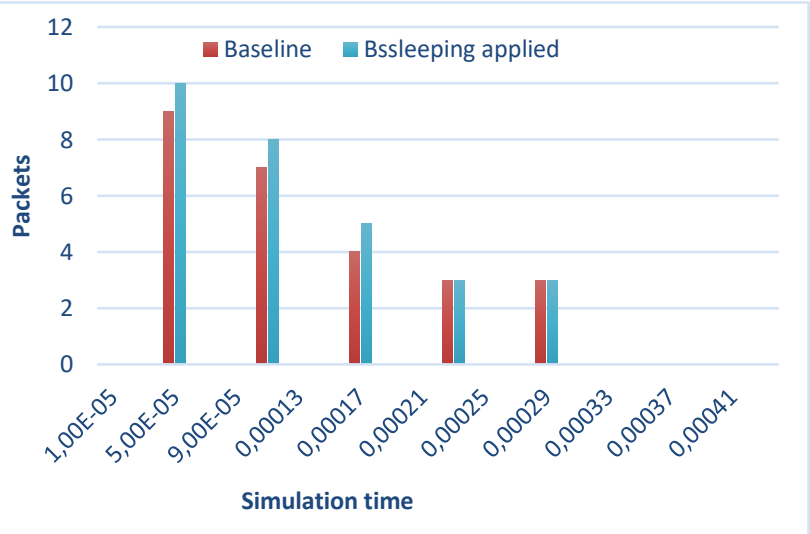

Fig. 10. Queue length at the main server 
The results show that the queue length was not affected by the BS sleeping algorithm. It can be observed that at the beginning of the simulation, the queue length was slightly long. This was however true for both cases. This can be explained by the fact that, at the beginning of the simulation, network elements do not operate at maximum capacity. Maximum capacity is achieved after a very short period after all the network elements have successfully undergone an initialisation phase. It can be observed that after 290 microseconds, the queues at the server completely disappear. The results show that the queue length is not affected by the BS sleeping algorithm. This means that QoS of the network is not compromised.

\section{Channel Quality Indicator (CQI)}

CQI is information sent by UEs to BSs pertaining to how the UEs perceive the channels in terms of quality. For LTE and LTE-A systems, values between 0 and 15 are used with 15 representing the best channel quality. In the network, CQI is used by BSs to allocate different modulation schemes and the adaptation of scheduling. Before application of the BS sleeping algorithm, CQIs from 17 UEs were collected and collected again after some micro BSs were put to sleep. Table IV shows the results and Figure 11 shows the plotted results. The results show that the CQI remained almost unchanged meaning that QoS was not compromised by the BS sleeping scheme.

TABLE IV

CQIS OF 17 UES BEFORE AND AFTER SOME BSS WERE SWITCHED OFF

\begin{tabular}{|l|l|l|}
\hline UE & $\begin{array}{l}\text { CQIs of 17 UEs before some } \\
\text { BSs were switched OFF }\end{array}$ & $\begin{array}{l}\text { CQIs of 17 UEs after some } \\
\text { BSs were switched OFF }\end{array}$ \\
\hline 1 & 8 & 8 \\
\hline 2 & 15 & 14 \\
\hline 3 & 15 & 14 \\
\hline 4 & 15 & 15 \\
\hline 5 & 14 & 14 \\
\hline 6 & 15 & 15 \\
\hline 7 & 15 & 15 \\
\hline 8 & 15 & 15 \\
\hline 9 & 15 & 15 \\
\hline 10 & 15 & 15 \\
\hline 11 & 14 & 12 \\
\hline 12 & 12 & 13 \\
\hline 13 & 12 & 13 \\
\hline 14 & 15 & 14 \\
\hline 15 & 12 & 12 \\
\hline 16 & 13 & 15 \\
\hline 17 & 4 & 7 \\
\hline
\end{tabular}

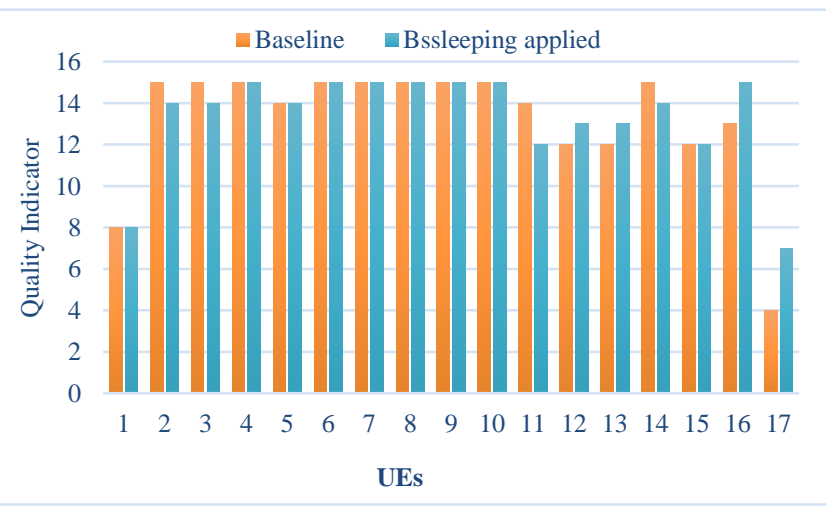

Fig. 11. CQIs for 17 UEs
It can also be observed that for some UEs, the CQI actually increased. This could have been caused by reduced interference levels since interference is reduced when some BSs are switched OFF.

\section{E. Sum Throughputs}

The UEs' sum throughput refers to packets that are successfully delivered to individual UEs in a network within a specified time. This performance metric is very important in determining the QoS of a network. To further evaluate the performance of the proposed scheme, UEs throughputs for 17 UEs were measured before and after introduction of the BS sleeping scheme. Table V shows the results obtained before and after application of the BS sleeping algorithm. The results are plotted in Figure 12.

TABLE V

UE SUM THROUGHPUTS BEFORE AND AFTER SwITCHING OFF SOME BSS

\begin{tabular}{|l|l|l|}
\hline UE & $\begin{array}{l}\text { Sum throughput before some } \\
\text { BSs were switched OFF }\end{array}$ & $\begin{array}{l}\text { Sum throughput after some BSs } \\
\text { were switched OFF }\end{array}$ \\
\hline 1 & 40 & 39 \\
\hline 2 & 40 & 40 \\
\hline 3 & 38 & 38 \\
\hline 4 & 37 & 37 \\
\hline 5 & 40 & 39 \\
\hline 6 & 40 & 40 \\
\hline 7 & 36 & 36 \\
\hline 8 & 38 & 37 \\
\hline 9 & 38 & 38 \\
\hline 10 & 40 & 40 \\
\hline 11 & 36 & 36 \\
\hline 12 & 39 & 40 \\
\hline 13 & 38 & 38 \\
\hline 14 & 37 & 37 \\
\hline 15 & 36 & 36 \\
\hline 16 & 40 & 40 \\
\hline 17 & 40 & 40 \\
\hline
\end{tabular}

Baseline $\quad$ Bssleeping applied

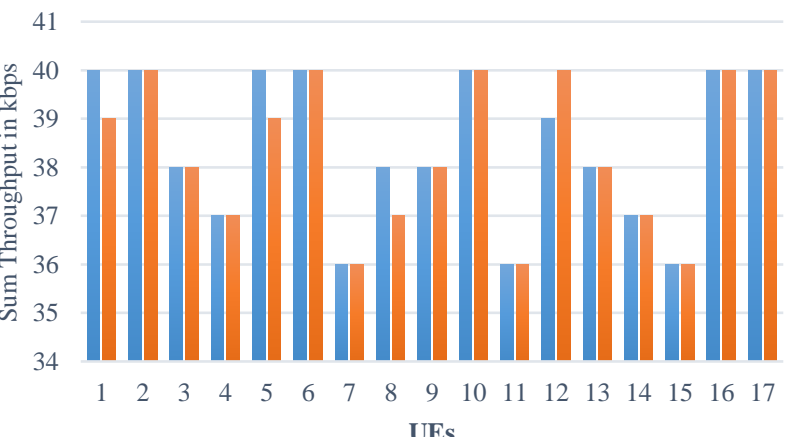

UEs

Fig. 12. UEs' sum throughputs

It can be seen that slightly over $75 \%$ of the UE's throughputs remained the same. This could have been achieved because the UEs could have been transferred to BSs that were not handling heavy traffic. Some UEs even showed an increased throughput. This can happen because when some BSs are switched OFF, interference levels are likely to decrease and this can push up UEs' throughputs. It can be deduced that the QoS of the network was not affected by the introduction of the BS sleeping scheme since the UEs' did not change. 


\section{F. Power consumption}

With SimuLTE 0.9.1, it is possible to measure real time network energy consumption. It was important to determine whether the scheme resulted in any energy saving and if there was, to quantify the energy saving. Table VI presents the results obtained. Figure 13 shows a linear reduction of total energy consumed in the network as the number of micro BSs being switched OFF increases. For this simulation the maximum number of micro BSs was seven and this represented a maximum energy saving of slightly more than 100 Joules. This might appear a very small energy saving but it can amount to high energy savings where large networks that are far denser are considered.

TABLE VI

TOTAL TRANSMITTED ENERGY AS BSS ARE SWITCHED OFF

\begin{tabular}{|c|c|}
\hline $\begin{array}{l}\text { Number of Micro BSs } \\
\text { switched OFF }\end{array}$ & $\begin{array}{l}\text { Total transmitted energy by all BSs in a sector } \\
\text { (in Joules) }\end{array}$ \\
\hline 1 & 403 \\
\hline 2 & 386 \\
\hline 3 & 369 \\
\hline 4 & 352 \\
\hline 5 & 335 \\
\hline 6 & 318 \\
\hline 7 & 301 \\
\hline
\end{tabular}

Fig. 13. Energy consumed versus number of BSs switched OFF

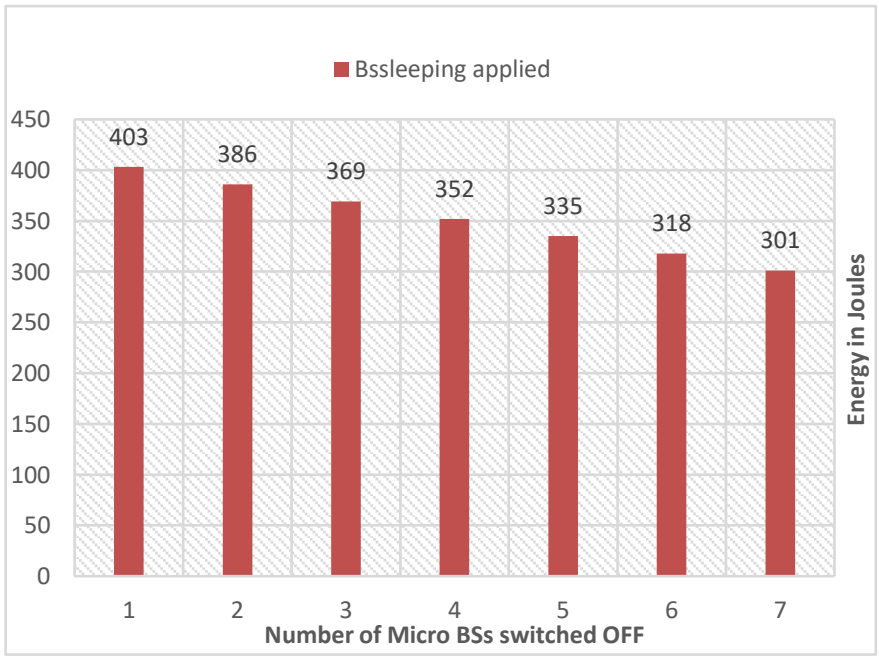

\section{CONCLUSIONS}

In this paper a BS sleeping scheme has been presented. The proposed scheme identifies micro BSs with very little traffic, checks whether neighbouring macro BSs can handle the extra traffic and puts the micro BSs to sleep. This scheme is designed for cellular networks that need to upgrade their networks to meet the ever growing cellular traffic. The scheme involves setting up micro BSs in areas already covered by macro BSs and are regularly frequented by high volumes of users like shopping malls. The micro BSs address capacity issues and the existing macro cells continue to provide uninterrupted cellular coverage. As traffic decreases, the scheme puts the micro BSs into sleep to save energy. Before micro BSs are put to sleep, the UEs are handed over to macro BSs that can best serve them. The current data rates of the UEs and the current traffic loads being handled by the macro BSs are considered to ensure an acceptable QoS is maintained.

\section{A. Implications of the research}

The research showed that it is possible to put to sleep lightly loaded micro BSs in a network and handover the traffic to accommodating macro BSs without affecting the QoS of the network. The scheme resulted in energy saving. Network operators are likely to embrace the scheme so as to reduce the operational expenditure. This would then make their profits match their profits. The scheme can also assist network operators in sprucing up their corporate image by use of green technologies that are energy efficient leading to reduced carbon dioxide emissions.

\section{B. Applications of the research}

The research work has managed to put together literature on Green Cellular Networks that researchers may find useful when designing energy efficient networks for future networks. The proposed scheme is likely to be used by established cellular networks on upgrading their networks to cater for the growing capacity

\section{Recommendations for further work}

SimuLTE 0.9.1 was used together with INET 2.3.0 and OMNeT++ v4.6 for the simulation of models used in the testing of the proposed BS sleeping scheme. It was therefore only possible to simulate LTE-A networks because SimuLTE 0.9.1 can only simulate up to and including LTE-A. LTE-A is a bridge between $4 \mathrm{G}$ and $5 \mathrm{G}$. This means the results that were posted by the BS sleeping technology when simulated using LTEAdvanced, might slightly change if it's used on true 5G. 5G will be handling Internet of Things traffic and it is strongly recommended that the technology be simulated using a simulator that can generate true $5 \mathrm{G}$ traffic. Outside cells were used in this project to generate interference but this can be different to the interference that is experienced in $5 \mathrm{G}$ networks.

\section{REFERENCES}

[1] Cisco. (2016, Feb). Cisco visual networking index: Global mobile data traffic forecast update, 2015-2020. [Online]. Available:http://www.cisco.com/c/en/us/solutions/collater al/service-provider/visual-networking-index-vni/mobilewhite-paper-c11-520862.html

[2] GSMA, "GSMA Global Mobile Economy Report, 2015".

[3] Nuclear Energy Agency, "Nuclear Energy: Combating climate change," Dec. 2015

[4] S.C Herring, J. Kossin, M. Hoerling, P. Stott and T. Peterson, "Explaining extreme events of 2014: from a climate perspective." Special Supplement to the Bulletin of the American Metrological Society, vol. 56 Issue no. 12, Dec. 2015.

[5] Monster twin tornadoes strike Pilger, Nebraska, Jun. 17 2014, [Online]. Available : $\quad$ www.earthsky.org/earth/monster-twin-tornadoes-strikepilger-nebrsaka

[6] A. Bousia, A. Antonopoulos, L. Alonso \& C. Verikoukis, “"'Green” Distance-aware base station sleeping algorithm in LTE- advanced," in IEEE International Conference on Communications, Ottawa, Canada, Jun. 2012, pp.1347-1351.

[7] Z. Niu, Y. Wu, J. Gong \& Z. Yang, "Cell zooming for cost-efficient Green Cellular Networks," IEEE Communications Magazine, vol. 48, Issue 11, pp. 74-79, Nov. 2013.

[8] S. Zhou, J. Gong \& Z. Niu, "Sleep control for base stations powered by heterogeneous energy sources," in International Conference on ICT Convergence (ICTC), Jeju Island, South Korea, Oct. 2013, pp. 666-670.

[9] K. Son \& B. Krishnamachari, "Dynamic base station switching-on/OFF strategies for green cellular networks," IEEE transactions on wireless communications, vol. 12, Issue 5, pp. 2126-2136, May 2013. 
[10] I. Aydin, H. Yanikomeroglud \& U. Aygolu, "User-aware switch-OFF algorithms," in $11^{\text {th }}$ International Wireless Communications and Mobile Computing Conference (IWCMC), Dubrovnik, Croatia, Aug. 2015, pp. 112-117.

[11] Wireless Technology: Breaking the limits of the TCO Reduction in Mobile Networks, Frost and Sullivan, 2011 White paper.

[12] M.H Asharif, R. Nordin \& M. Ishmail. "A survey of green radio communication networks: Techniques and recent advances," Journal of computer networks and communications,2013, vol. 2013, Article 45389 , 13 pages, Hindawi Publishing Corporation, Nov. 2013.

[13] K. Davaslioglu and E. Ayanoglu, "Quantifying potential energy efficiency gain in Green Wireless Networks," IEEE Communications Surveys and Tutorials, vol. 16, pp. 2065-2091, Fourth Quarter, 2014.

[14] A. Conte, A. Feki, L. Chiaraviglio, D. Ciullo, M. Meo \& M. A Masan, "Cell wilting and blossoming for energy efficiency," Wireless Communications, IEEE, vol. 18, Issue 5, pp. 50-57, Oct. 2013.
[15] W. Mwashita and M.O Odhiambo, "A survey on Base station sleeping technologies for Green Cellular Networks," International Conference on Wireless Networks, Las Vegas, USA, Jul 2015, pp. 227-233.

[16] W. Mwashita and M.O Odhiambo, "Designing and analysing a technique to switch ON/OFF Base Stations for Green Cellular networks," International Journal of Research in Engineering and Technology, vol. 4, Issue 07, Jul 2015, pp. 322-328.

[17] P. Dini, M. Miozzo, N. Bui \& N. Baldo, "A model to analyze the energy savings of base station sleeping in LTE HetNets," in IEEE International Conference on Green computing and communications and IEEE Internet of Things and IEEE Cyber, Physical and Social Computing, Beijing, China, Aug. 2013, pp. 1375-1380.

[18] OMNeT++, An object oriented modular discrete event network simulation framework, 2016. [Online]. Available: https://omnetpp.org

[19] SimuLTE. LTE User Plane Simulation Model for INET \& OMNeT++, [Online]. Available: www.simulte.com

[20] INET Framework. An open source model suite for wired and wireless mobile networks, Available: https://inet.omnetpp.org 